\title{
ON THE STABILITY OF TWO-DIMENSIONAL PARALLEL FLOWS
}

\section{PART I.-GENERAL THEORY*}

\author{
BY \\ C. C. LIN \\ Guggenheim Laboratory, California Institute of Technology
}

1. Introduction. The study of the stability of laminar motion and its transition to turbulence dates back to the time of Helmholtz and Reynolds [46], and had already attracted great attention at the end of the last century. ${ }^{* *}$ Since that time, the subject has not only become a major problem for workers in hydrodynamics, but has also attracted the attention of people like Lord Rayleigh [43-45], Lord Kelvin [20-21], Lorentz [29], Sommerfeld [58], and Heisenberg [14], whose chief interest is not limited to the study of mechanics. Although numerous contributions have since been made, the subject has remained one of considerable dispute, as can be seen from the two general lectures given by Taylor [70] and by Synge [63] as late as 1938. Still more recently, there appeared the work of Görtler $[8,9]$ and of Thomas [71].

Most of the work on the stability of laminar motions has the following final aims.

1) The first aim is to determine whether a given flow (or a given class of flows) is ultimately unstable for sufficiently large Reynolds numbers. For this purpose, it is desirable to obtain some simple general criterion which will give a rapid classification of velocity profiles according to their stability.

2) The second purpose is to determine the minimum critical Reynolds number at which instability begins. It is often easier to find sufficient conditions for stability than to find the condition for passage from stability to instability.

3) Finally, we want to understand the physical mechanism underlying the phenomena by giving theoretical interpretations and experimental confirmations of the results obtained from mathematical analysis.

Although numerous attempts have been made in these directions, especially for the apparently simplest cases of parallel flows in two dimensions, our knowledge is still very meagre. The classical case of plane Pouiseuilla motion has remained an unsettled problem. $\dagger$ and no satisfactory general results have been reached regarding the stability of a real fluid. The best-known general criterion is that of Rayleigh (1880) and Tollmien [74], classifying profiles according to the occurrence of a flex $\dagger \dagger$ with respect to the stability of a fluid at infinite Reynolds numbers. However, the significance of their results has been too much exaggerated and often misunderstood, and no physical interpretation has ever been given. The present work offers such an interpretation, but also shows that the results can only give some indication regarding

\footnotetext{
* Received March 3, 1945. An abstract of this paper has already appeared under the same title [27].

** In 1888, the problem was proposed by Rayleigh and Stokes as the subject for the Adams Prize Essay. Cf. p. 321 of Ref. [21], and also the footnote on p. 267 of Ref. [44].

$\dagger$ Cf. Synge's lecture [63].

†† Following Professors Frank Morley and H. Bateman, we shall use the word "flex" for "point of inflection."
} 
the instability of a real (viscous) fluid. This will be discussed in more detail below.

The chief aim of the present work is to try to answer the three questions mentioned above for two-dimensional parallel flows. This work is divided into three parts. Part I (the present paper) deals with the general mathematical theory, with particular emphasis on attempting to clarify the mathematical difficulties involved in the solution of the equation of stability. Part II deals with the stability problem in an inviscid fluid (infinite Reynolds numbers). Part III deals with the problem in a real fluid. The following results have been obtained.

1) It is shown that all velocity distributions of the symmetrical type and of the boundary-layer type are unstable for sufficiently large (but finite) values of the Reynolds number (Part III). The plane Poiseuille motion is included as a special case.

2) A simple approximate method is obtained by which one can calculate the minimum Reynolds number marking the beginning of instability with very little numerical labor (Part III).

3) The tendency toward instability of a profile with a point of inflection is interpreted by considering the distribution of vorticity (Part II). The effect of viscosity is considered as diffusing the disturbance from the "critical layer" inside the fluid and from the solid boundary. A very simple quantity is thereby derived which serves as a measure of the effect of viscosity (Part III). This can also be easily connected with the general mathematical theory.

As numerical examples, we have worked out the curve of neutral stability for the Poiseuille case and the Blasius case. Comparisons with existing results are discussed (Part III). The relation between instability and transition to turbulence is also discussed in Part III of this work.

Since some of the present results differ markedly from customary beliefs, it is necessary to trace the history of the existing lines of thought in order to give proper recognition to earlier ideas and results used in the present work, and to analyze all the results in disagreement with present conclusions. This requires the repetition of some known results when they fall into the present line of treatment. The review of literature is not intended to be exhaustive; only the necessary references are cited. A more complete bibliography up to 1932 has been given by Bateman [2].

2. Historical survey of existing theories. There seem to be two schools of thought in regard to the cause of transition from steady to turbulent conditions. One school contends that transition is due to a definite instability of the flow, i.e., to a condition in which infinitesimal disturbances grow exponentially. The second school regards the motion in most cases as definitely stable for infinitesimal disturbances but liable to be made turbulent by suitable disturbances of finite magnitude or by a large enough pressure gradient. Both schools, however, generally agree that the fluid can be considered as incompressible and that its motion is governed by the NavierStokes equations of motion. Since the agreement between theory and experiment has not been very satisfactory, it has also been proposed that the cause of transition must be traced back to the effect of compressibility or to the possible failure of the Navier-Stokes equations. The present work tends to confirm the simplest point of view that the motion in most cases is definitely unstable for infinitesimal disturbances governed by the Navier-Stokes equations for an incompressible fluid.

The theory of finite disturbances dates back to Reynolds [46] and Kelvin [21]. 
It was developed by Schiller, Taylor and others.* Mathematical investigations of such finite disturbances are mainly based on considerations of energy or of the square of the vorticity of the disturbance, because the solution of the non-linear equations satisfied by the disturbance is extremely difficult. At the end of Part III we shall briefly discuss this line of thought together with the results of the present paper. For more details, the reader is referred to the lecture of Taylor [70] and the papers of Synge [62] and Thomas [71].

For small disturbances, positive definite integrals of the energy and vorticity of the disturbance have been extensively used. These considerations have been discussed by Orr [37], Lorentz [29], von Kármán [18], Synge [63, 64] and others. For excellent accounts of this phase of the theory, the reader is referred to the works of Noether [35], von Kármán [18], Prandtl [42], and Synge [64]. Additional references are cited at the end of this paper. As is now well-known, this method can only give sufficient conditions for stability. Also, since all disturbances are usually allowed, including those which do not satisfy the hydrodynamic equations of motion, a larger viscous decay is required to insure stability than when these disturbances are excluded. Consequently, the limit of stability is always found to be much lower than that indicated by experiment. However, from these considerations, Synge [63] has arrived at a very convenient form of a sufficient condition for the stability of twodimensional parallel flows with respect to two-dimensional disturbances. This will be found very useful for the discussions in Part III.

To get more concrete results, we have to solve the linearized equations satisfied by the disturbance. The most successful case appeared to be Taylor's treatment of Couette flow [67] between concentric cylinders. His work was verified by the experiments carried out by himself $[67,69]$ and by others [28]. A rigorous mathematical investigation in this connection was made by Faxon [4]. In fact, it is now known that his analysis is a typical case of the stability of a fluid motion where the centrifugal force plays a dominant part. Such cases were first considered by Lord Rayleigh [45], who gave a condition for the stability of an inviscid fluid. Mathematical proof of a sufficient condition of stability of Couette flow was recently given by Synge [65]. Extension of Taylor's work to the boundary layer over a curved wall was carried out by Görtler $[8,9]$, who used numerical methods successfully.

While the investigation of curved flows was uneventful, the investigation of axially symmetrical flows was not extensive. The Poiseuille flow in a circular pipe was studied by Sexl [55] with a conclusion of stability. Prandtl [42] gave some discussions of the possible cause of instability in his article in the book "Aerodynamic Theory," edited by Durand.

The most extensive discussion of hydrodynamic stability seems to be the treatment of parallel flows by attempting to solve the eigen-value problem associated with the linearized equations governing the disturbance. This line of development can be easily traced in the work of Helmholtz, Lord Rayleigh [43, 44], Orr [37], Sommerfeld [58], von Mises [31, 32], Hopf [16], Prandtl [41], Tietjens [72], Heisenberg [14], Tollmien [73-75], and Schlichting [52-54]. Other contributions are those of Noether [36], Solberg [57], Southwell [59], Squire [60], Goldstein [6], Pekeris [39, 40], Synge [61-65] and Langer [25].

* See Taylor's lecture [70] for references to the works on finite disturbances. 
For convenience, the theory deals with two-dimensional wavy disturbances propagated along the direction of the main flow. Squire [60] has shown that three-dimensional wavy disturbances are more stable than two-dimensional ones. However, Prandtl still mentions the possibility of greater instability of three-dimensional disturbances in his article [42] appearing after Squire's paper.

The first study of two-dimensional hydrodynamic stability seems to have been made by Helmholtz. He proved the instability of wavy disturbances over the surface of discontinuity of two parallel streams of different velocities. Later, Rayleigh [43] realized that Helmholtz's approximation was not good enough to bring out the main features of a flow with continuous velocity distributions. He therefore made an im-

(a)

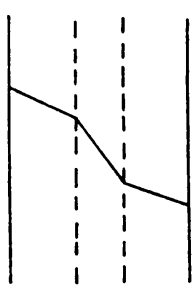

(b)

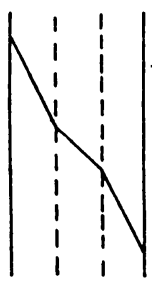

(c)

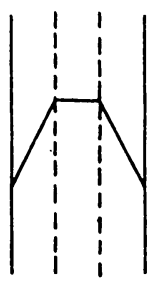

FIG. 1. Broken profiles investigated by Lord Rayleigh. Case

(a) may be unstable; the other two cases are stable proved approximation consisting of several linear profiles joined up continuously. The vorticity distribution then has constant values in several layers, but has a discontinuity in passing from one layer to another. Investigations with continuous vorticity distributions were also made. Rayleigh's work was mainly concerned with an inviscid fluid. Two main results were obtained. The first is that instabilit! (in an inviscid fluid) can only occur with velocity distributions having a point of inflection It is usually believed that Lord Rayleigh has also proved that damped disturbances call also only occur with such profiles. The possibility of a disturbance for a profile without a flex then becomes a paradox [5]. Actually, Rayleigh's proof does not lead to such a conclusion. This point will be more fully discussed in $\$ 5$ and Part II. Rayleigh's second result is obtained from the analysis of broken linear profiles; it substantiates the first result by demonstrating definite instability of broken linear velocity distributions of the type shown in Fig. 1(a), and only stability in the other cases. Rayleigh [43] supported his result by obtaining the condition determining stability in the approximate form

$$
\int_{y_{1}}^{y_{2}}(w-c)^{-2} d y=0,
$$

where $w(y)$ is the velocity distribution, $y_{1}$ and $y_{2}$ are the coordinates of the solid boundaries, and $c$ is a constant the real part of which represents the wave velocity and the imaginary part of which gives damping or amplification.

Meanwhile, the exact analysis of linear velocity distributions including the effect of viscosity was given by von Mises [31,32], and Hopf [16] and was also studied by Rayleigh [44]. The results indicate only stability. Prandtl and Tietjens [72] applied Rayleigh's method of approximation to the stability of the boundary layer, taking account of the effect of viscosity. In such an approximation, the inner friction layer mentioned above $(\$ 1)$ for continuous vorticity distributions is left out. The result of Tietjens did not give a minimum critical Reynolds number.

It was Heisenberg [14] who first successfully studied the stability of a variable 
continuous vorticity distribution. As a particular example, he demonstrated that the plane Poiseuille flow was unstable for sufficiently large Reynolds numbers. Also, using the same equation (2.1) with which Rayleigh supported his approximation with linear profiles, Heisenberg pointed out the fallacy in Rayleigh's method. The essential point is that the corners in the velocity profile introduce extraneous roots of the above equation for $c$. Consequently, the results of this type of analysis depend upon the manner in which the velocity distribution is approximated.

Heisenberg's numerical computation was, however, incomplete and very rough, and his theory was not generally accepted. Better known are the results of Tollmien and Schlichting. They studied the cases of Blasius [73] and plane Couette flow [48], using Heisenberg's theory essentially. The former case was pursued very much in detail. For the latter case, Schlichting followed the idea of Prandtl, asserting that the instability may be attributed to the initial unsteady distribution prior to the formation of the linear profile. Indeed, the same kind of idea was also suggested by Prandtl to account for the instability of Poiseuille flow by ascribing it to the entrance section where the profile is not yet parabolic [41]. This problem will be discussed in some detail later ( $\$ 14$, Part III).

For an inviscid fluid, Tollmien has also proved the instability of boundary-layer and symmetrical profiles with a point of inflection [74]. For a viscous fluid, the present investigation shows that instability depends upon the general type of these profiles rather than on the appearance of the point of inflection. The inner friction laver plays a dominant role in determining the instability. Attempts to interpret this point physically are given by Prandtl [42] and in the present paper.

3. General formulation of the problem. We shall now formulate the problem of the stability of two-dimensional parallel flows mathematically In the first place. we note that if the steady motion is strictly two-dimensional and parallel, the velocity distribution must be either linear or parabolic (if body forces are absent). We then have one of the following: 1) the plane Couette flows; 2) the plane Poiseuille flow; 3 ) a combination of these two flows. The problem would then be very restricted.

However, there are a large number of cases where the flow is essentially parallel to one direction. These are the cases where the boundary-layer consideration is permissible. The following are important special cases belonging to this class: 4 ) inlet flow between parallel walls, flow in a slightly convergent or divergent channel; 5) flow along a flat plate; 6 ) wake behind a cylindrical body, jet from a narrow slit. Whether these flows can be properly considered as belonging to the same class as the above three is a question of some controversy. Taylor has criticized Tollmien's work with the boundary layer on this ground [70]. In the Appendix to Part III of this work, we shall try to demonstrate that this treatment is generally permissible, but that the interpretation of the results must be taken up with care. A discussion of Tollmien's work will also be found there.

In considering the stability of the main flow, we superpose upon it a hydrodynamically possible small disturbance, and consider its behavior. The disturbance is small in the sense that the inertia forces corresponding to the disturbance alone are negligible and that its behavior is unaltered when its amplitude is (say) doubled or halved. It is then simplest to consider separate harmonic components with respect to time, which may be damped, neutral, or self-excited. By considering disturbances which are also spacially periodic both in the direction of flow and in the direction 
perpendicular to the plane of symmetry of the main motion, Squire [60] was able to show that two-dimensional disturbances are less stable than three-dimensional disturbances. Hence, important features of the stability problem can be obtained by considering two-dimensional disturbances alone. This is an essential difference between the stability of a parallel flow and of a curved flow. In the latter case, threedimensional disturbances are of utmost importance.

The consideration of periodic disturbances alone is again a question of some controversy. Justification has been attempted and objection has been raised. We shall see later that at least the difficulties raised are chiefly caused by a misinterpretation of the mathematical results.

Admitting that we can consider two-dimensional disturbances alone, we have a much simplified physical picture at hand. If the effect of viscosity is negligible, we have the well-known fact of conservation of vorticity for two-dimensional motions. Actually, the stability problem is found to depend both on the inertia forces and on the viscous forces. However, the effect of viscosity is also well-known to be one of diffusion of vorticity. Thus, important results can be expected from considerations of vorticity transfer.

Let us now proceed with the mathematical formulation of the problem. We shall give a complete derivation of the stability equations so that we can see how to settle the disputes about the approximations in considering velocity distributions of the boundary-layer type.

Admitting Squire's work as a proper indication that only two-dimensional disturbances need be considered, we may conveniently consider the equation of vorticity

$$
\Delta \psi_{\imath}+\psi_{\nu} \Delta \psi_{x}-\psi_{x} \Delta \psi_{\nu}=\nu \Delta \Delta \psi
$$

with the velocity components

and the vorticity

$$
u=\psi_{\nu}=\frac{\partial \psi}{\partial y}, \quad v=-\psi_{x}=-\frac{\partial \psi}{\partial x},
$$

$$
\zeta=\frac{\partial v}{\partial x}-\frac{\partial u}{\partial y}=-\left(\psi_{x x}+\psi_{y y}\right)=-\Delta \psi .
$$

As usual, $\nu$ is the kinematical viscosity. We may add that Squire's original proof was intended for flow bounded between two parallel walls. There is no difficulty in seeing that the proof holds also for a fluid extending to infinity, ${ }^{*}$ because the boundary conditions for the disturbance are essentially the same.

Let us put

$$
\psi=\Psi(x, y)+\psi^{\prime}(x, y, t),
$$

where $\Psi(x, y)$ represents the steady main flow and $\psi^{\prime}(x, y, t)$ represents the disturbance. Main flows which vary but slowly with time can also be treated this way, but we shall restrict ourselves to steady flows in order to fix our ideas.

If we substitute (3.4) into (3.1) the terms corresponding to the main flow cancel out. If we then drop the terms quadratic in $\psi^{\prime}(x, y, t)$ and its derivatives, we have the equation

$$
\Delta \psi_{t}^{\prime}+\Psi_{y} \Delta \psi_{x}^{\prime}-\Psi_{x} \Delta \psi_{y}^{\prime}+\psi_{y}^{\prime} \Delta \Psi_{x}-\psi_{x}^{\prime} \Delta \Psi_{y}=\nu \Delta \Delta \psi^{\prime} .
$$

* Cf. Ref. [15]. 
We shall now assume the flow to be essentially parallel to the $x$-axis. Using the boundary-layer approximation, we should drop the $x$-derivative of any quantity connected with the main flow compared with its $y$-derivative. But for the disturbance we would expect $\psi_{x}^{\prime}$ and $\psi_{y}^{\prime}$ to be of the same order of magnitude. This will be verified $a$ posteriori in the specific examples. Further discussions will be found in the Appendix to Part III. With these considerations, (3.5) reduces to

$$
\Delta \psi_{i}^{\prime}+\Psi_{y} \Delta \psi_{x}^{\prime}-\psi_{x}^{\prime} \frac{\partial^{3} \Psi}{\partial y^{3}}=\nu \Delta \Delta \psi^{\prime} .
$$

Now we shall make an approximation of the same order by taking for $w=\Psi_{v}$ and $\partial^{2} w / \partial y^{2}=\partial^{3} \Psi / \partial y^{3}$ their local values at a given value $x_{0}$ of $x$. Then we may write

$$
\Delta \psi_{t}^{\prime}+w(y) \Delta \psi_{x}^{\prime}-w^{\prime \prime}(y) \psi_{x}^{\prime}=\nu \Delta \Delta \psi^{\prime} .
$$

For the boundary conditions, we shall also consider the local boundaries. The problem is then essentially simplified, and can be treated similarly to plane Couette and Poiseuille flows. We consider a main flow between two parallel planes $y=y_{1}$ and $y=y_{2}$ with a more or less arbitrary distribution of velocity $w(y)$. Then the disturbance $\psi^{\prime}(x, y, t)$ must be found as a solution of (3.7) satisfying the conditions $u^{\prime}=v^{\prime}=0$ over the boundaries.

The usual way of dealing with the solution of (3.7) subject to given boundary conditions is to consider periodic disturbances. We shall refer all velocities to a characteristic velocity $U$ and all lengths to a characteristic length $l$, and define the Reynolds number $R=U l / \nu$. The two-dimensional periodic disturbance of a field of flow in which the main flow is $w(y)$ may be represented by the stream function $\psi^{\prime}=\phi(y) e^{i \alpha(x-c t)}$, and the linearized differential equation for $\phi(y)$ is

$$
(w-c)\left(\phi^{\prime \prime}-\alpha^{2} \phi\right)-w^{\prime \prime} \phi=-\frac{i}{\alpha R}\left(\phi^{i v}-2 \alpha^{2} \phi^{\prime \prime}+\alpha^{4} \phi\right),
$$

as can be easily obtained from (3.7). We shall take $\alpha$ always real and positive, while $c$ may be complex; thus,

$$
c=c_{r}+i c_{i} .
$$

To fix our ideas about the boundary conditions, let us consider a flow between the planes $y=y_{1}$ and $y=y_{2}$. The equation (3.8) is then to be solved under the boundary conditions

$$
\phi\left(y_{1}\right)=0, \quad \phi\left(y_{2}\right)=0, \quad \phi^{\prime}\left(y_{1}\right)=0, \quad \phi^{\prime}\left(y_{2}\right)=0 .
$$

Let us now forget about the physical problem and consider the differential equation (3.8) as a linear differential equation of the fourth order in the complex $y$-plane. To be sure, the function $w(y)$ is defined only for real values of $y$ between $y_{1}$ and $y_{2}$. We can of course, consider it as defined for other values of $y$ by analytical continuation. We shall assume that the function thus defined is holomorphic in every finite region with which we shall be concerned. The equation (3.8) then has every point in the region under consideration as a regular point, and its coefficients are also entire functions of the parameters $c, \alpha$, and $\alpha R$ (regarded as complex variables). By a wellknown theorem in the theory of differential equations, there exists a fundamental 
system of four solutions of (3.8) which are analytic functions of the variable $y$ and of the parameters $c, \alpha$, and $\alpha R$, being in fact entire functions of the parameters. The consequences of these simple general analytical considerations appear to have escaped serious attention from earlier investigators. In $\$ \S 4,5$ of this paper, we shall find this type of consideration very important in settling the controversies about the question of convergence of the series used in the actual solution of equations (3.8) and (3.14).

Let us denote the above-mentioned system of solutions of (3.8) by $\phi_{1}(y), \phi_{2}(y)$, $\phi_{3}(y), \phi_{4}(y)$, the dependence upon the parameters $c, \alpha, \alpha R$ being understood. The conditions (3.10) then give rise to the secular equation

$$
F(c, \alpha, \alpha R)=\left|\begin{array}{llll}
\phi_{1}\left(y_{1}\right) & \phi_{2}\left(y_{1}\right) & \phi_{3}\left(y_{1}\right) & \phi_{4}\left(y_{1}\right) \\
\phi_{1}\left(y_{2}\right) & \phi_{2}\left(y_{2}\right) & \phi_{3}\left(y_{2}\right) & \phi_{4}\left(y_{2}\right) \\
\phi_{1}^{\prime}\left(y_{1}\right) & \phi_{2}^{\prime}\left(y_{1}\right) & \phi_{3}^{\prime}\left(y_{1}\right) & \phi_{4}^{\prime}\left(y_{1}\right) \\
\phi_{1}^{\prime}\left(y_{2}\right) & \phi_{2}^{\prime}\left(y_{2}\right) & \phi_{3}^{\prime}\left(y_{2}\right) & \phi_{4}^{\prime}\left(y_{2}\right)
\end{array}\right|=0 .
$$

Since the function $F(c, \alpha, \alpha R)$ is an entire function of the variables $c, \alpha, \alpha R$, we may solve for $c$, obtaining

$$
c=c(\alpha, R) .
$$

There may be several branches of the solution, or there may be none as in the case when $F(c, \alpha, \alpha R)$ is (say) $\exp (\alpha R c)$. In general, we would expect the solution to be unique, or we may consider only one branch of the solution.

Since $\alpha$ and $R$ are later taken to be real and positive, it is convenient to separate (3.12) into its real and imaginary parts. Thus,

$$
c_{r}=c_{r}(\alpha, R), \quad c_{i}=c_{i}(\alpha, R) .
$$

It is customary to plot curves of constant $c_{i}$ or $\alpha c_{i}$ in the $\alpha R$-plane. The curve $c_{i}=0$ gives the limit of stability.

We are particularly interested in the case when the Reynolds number is very large. The study of this case is complicated by the fact that the functions $\phi_{1}, \phi_{2}, \phi_{3}, \phi_{4}$ involved have essential singularities at the infinite point of the $\alpha R$-plane. From the differential equation (3.8) itself, we see that when $\alpha R \rightarrow \infty$, we have the equation

$$
(w-c)\left(\phi^{\prime \prime}-\alpha^{2} \phi\right)-w^{\prime \prime} \phi=0,
$$

which is only of the second order. Thus, two solutions of (3.8) are lost. From detailed mathematical investigations, we shall find later that two linearly independent solutions of (3.8), say $\phi_{1}$ and $\phi_{2}$, will satisfy (3.14) in the limit of infinite $\alpha R$, except along certain straight lines through the point $w=c$. The other two linearly independent solutions $\phi_{3}$ and $\phi_{4}$ are highly oscillating for large $\alpha R$ and would therefore disapapear in the limit of infinite $\alpha R$. Furthermore, we shall see that $\phi_{3}$ and $\phi_{4}$ can be so chosen that if $\phi_{3}\left(y_{1}\right) \gg \phi_{4}\left(y_{1}\right)$, then $\phi_{3}\left(y_{2}\right) \ll \phi_{4}\left(y_{2}\right)$, with corresponding relations for their derivatives. It then appears plausible that the limiting form of (3.11) for infinite $\alpha R$ is

$$
\left|\begin{array}{ll}
\phi_{1}\left(y_{1}\right) & \phi_{2}\left(y_{1}\right) \\
\phi_{1}\left(y_{2}\right) & \phi_{2}\left(y_{2}\right)
\end{array}\right|=0,
$$

with $\phi_{1}(y), \phi_{2}(y)$ satisfying (3.14). 
The condition (3.15) states that we are looking for a solution $\phi(y)$ of (3.14) satisfy-

$$
\phi\left(y_{1}\right)=0, \quad \phi\left(y_{2}\right)=0,
$$

with the other two conditions of (3.10) relaxed. Physically, this means that we allow a slipping along the walls $y=y_{1}$ and $y=y_{2}$. For very large Reynolds numbers, only a very thin layer of fluid will stick to the solid, and we have naturally an apparent slipping. These points will be taken up again more carefully ( $\$ 6)$ after a thorough mathematical investigation of the solutions.

4. Solution of the equation of Orr and Sommerfeld by methods of successive approximation. The stability equation of Orr and Sommerfeld

$$
(w-c)\left(\phi^{\prime \prime}-\alpha^{2} \phi\right)-w^{\prime \prime} \phi=-\frac{i}{\alpha R}\left(\phi^{\mathrm{iv}}-2 \alpha^{2} \phi^{\prime \prime}+\alpha^{4} \phi\right)
$$

has a fundamental system of four solutions, which are analytic functions of $y$ (whereever $w(y)$ is analytic) and which are entire functions of $\alpha, c$, and $\alpha R$. In order to obtain useful solutions, it is usual to expand the solutions as power series of a suitable small parameter, say, $(\alpha R)^{-1}$. However, since $(\alpha R)^{-1}$ occurs with the highest derivative in (4.1), the study of such an expansion becomes very complicated. It will be done later.

a) Solution by convergent series. An alternative method* is to choose a small parameter $\epsilon$ related to $(\alpha R)^{-1}$ and first make a change of variable $\left(y_{0}\right.$ being an arbitrary point so far)

$$
y-y_{0}=\epsilon \eta, \quad \phi(y)=\chi(\eta),
$$

so that $(4.1)$ becomes

$$
(w-c)\left(\chi^{\prime \prime}-\alpha^{2} \epsilon^{2} \chi\right)-\epsilon^{2} w^{\prime \prime} \chi=-\frac{i}{\alpha R \epsilon^{2}}\left(\chi^{\mathrm{iv}}-2 \alpha^{2} \epsilon^{2} \chi^{\prime \prime}+\alpha^{4} \epsilon^{4} \chi\right),
$$

where

$$
\left.\begin{array}{rl}
w-c & =\left(w_{0}-c\right)+w_{0}^{\prime}(\epsilon \eta)+\frac{w_{0}^{\prime \prime}}{2 !}(\epsilon \eta)^{2}+\cdots, \\
w^{\prime \prime} & =w_{0}^{\prime \prime}+w_{0}^{\prime \prime \prime}(\epsilon \eta)+\frac{w_{0}}{2 !}(\epsilon \eta)^{2}+\cdots
\end{array}\right\}
$$

The solution is then obtained in the form

$$
\phi(y)=\chi(\eta)=\chi^{(0)}(\eta)+\epsilon \chi^{(1)}(\eta)+\epsilon^{2} \chi^{(2)}(\eta)+\cdots,
$$

and the differential equations for the approximations of successive orders can be obtained by substituting (4.4) and (4.5) into (4.3) and equating all the coefficients of the various powers of $\epsilon$ to zero.

If we take $y_{0}$ to be the point where $w=c$, the proper choice of the parameter $\epsilon$ is

$$
\epsilon=(\alpha R)^{-1 / 3} \text {. }
$$

The differential equations for the functions $\chi^{(0)}(\eta), \chi^{(1)}(\eta), \chi^{(2)}(\eta), \cdots$ are as follows:

$$
\left.\begin{array}{ll}
\epsilon^{0} ; & w_{0}^{\prime} \eta \chi^{(0) \prime \prime}+i \chi^{(0) \mathrm{iv}}=0, \\
\epsilon^{n} ; & w_{0}^{\prime} \eta \chi^{(n) \prime \prime}+i \chi^{(n) \mathrm{iv}}=L_{n-1}(\chi), \quad(n \geqq 1),
\end{array}\right\}
$$

* This method was first used by Heisenberg, loc. cit. [14], p. 588. 
where $L_{n-1}(\chi)$ is a linear combination of $\chi^{(0)}(\eta), \chi^{(1)}(\eta), \cdots, \chi^{(n-1)}(\eta)$ and their derivatives. In particular,

$$
L_{0}(\chi)=w_{0}^{\prime \prime}\left(\chi^{(0)}-\frac{1}{2} \eta^{2} \chi^{(0) \prime \prime}\right) .
$$

We note that the homogeneous part is the same for all the differential equations in (4.7). Hence, if we can solve for the first approximation, the rest can all be obtained by quadratures. Indeed, the first equation of (4.7) is Stokes' equation* for $\chi^{(0)}$ " , and its solution can be readily expressed in terms of Bessel functions of the order $1 / 3$. Thus, for the first equation of (4.7) we have the four particular integrals**

where

$$
\begin{aligned}
& \chi_{1}^{(0)}=\eta, \quad \chi_{3}^{(0)}=\int_{+\infty}^{\eta} d \eta \int_{+\infty}^{\eta} d \eta \eta^{1 / 2} H_{1 / 3}^{(1)}\left[\frac{2}{3}\left(i \alpha_{0} \eta\right)^{3 / 2}\right], \\
& \chi_{2}^{(0)}=1, \quad \chi_{4}^{(0)}=\int_{-\infty}^{\eta} d \eta \int_{-\infty}^{\eta} d \eta \eta^{1 / 2} H_{1 / 3}^{(2)}\left[\frac{2}{3}\left(i \alpha_{0} \eta\right)^{3 / 2}\right]
\end{aligned}
$$

$$
\alpha_{0}=\left(w_{0}^{\prime}\right)^{1 / 3} .
$$

The higher approximations are given by

$$
\begin{gathered}
\chi_{i}^{(n)}=\frac{\pi}{6} \int^{\eta} d \eta \int^{\eta} d \eta\left\{\chi_{4}^{(0) \prime \prime} \int^{\eta} d \eta \chi_{3}^{(0) \prime \prime} L_{n-1}(\chi)-\chi_{3}^{(0) \prime \prime} \int^{\eta} d \eta \chi_{4}^{(0) \prime \prime} L_{n-1}(\chi)\right\} . \\
(i=1,2,3,4) .
\end{gathered}
$$

These are the explicit formulae for finding the approximations of various orders. In actual calculations, only the initial approximation (4.9) is required. Furthermore, the series (4.5) is convergent provided $\epsilon$ is restricted so that the series (4.4) are convergent. For then the differential equation (4.3) for $\chi(\eta)$, when normalized, has analytic functions of the parameter $\epsilon$ as its coefficients. Hence, a fundamental system of its solutions consists of four analytic functions of $\epsilon$.

It should be mentioned that if $y_{0}$ is not taken at the particular point for which $w=c$, the proper parameter to be taken is $(\alpha R)^{-1 / 2}$ instead of $(\alpha R)^{-1 / 3}$. In this case, all the approximations can be expressed in terms of elementary transcendental functions. However, it is not found particularly advantageous to do so, because the study of "crossing substitution" ( $\$ 5$ ) would not be easy. Also, the method is then too much different from those used by earlier investigators to allow an easy comparison of the results.

b) Solution by asymptotic series. Although the previous method is theoretically

* Cf. the exact treatment of (4.1) by Hopf [16] and Rayleigh [44] for the case $w^{\prime \prime}=0$.

** Note that $\chi_{3}^{(0)}$ and $\chi_{i}^{(0)}$ and also $\chi_{i}^{(n)}$ have no branch point at $\eta=0$. The order of the solutions $\left\{\phi_{1}, \phi_{2}, \phi_{3}, \phi_{4}\right\}$ agrees with Tollmien's notation. They are $\left\{\phi_{3}, \phi_{4}, \phi_{1}, \phi_{2}\right\}$ in Heisenberg's notation. Heisenberg gave the solutions $\phi_{3}$ and $\phi_{4}$ in terms of Hankel functions in the form

$$
\phi_{i}=(w-c) \int \frac{1}{\eta} H_{2 / 3}^{(i)}\left[\frac{2}{3}\left(i \alpha_{0} \eta\right)^{3 / 2}\right] d \eta, \quad(j=1,2),
$$

(p. 289, and Eq. (19a) p. 591). It can be easily verified that these are the same as $\chi_{3,4}^{(0)}$ up to a constant factor $w_{0}^{\prime} \epsilon$ and to the proper order of approximation. Note that throughout Heisenberg's paper, $i$ is to be replaced by $-i$ in order to conform to our notation. This can be seen from a comparison of our Eq. (4.1) with his Eq. (7a). The difference arises from a difference of notation in the stream function $\psi^{\prime}(x, y, t)$. 
complete, it is usually more convenient to use asymptotic series for numerical purposes, particularly in dealing with boundary-value problems. Heisenberg has given two asymptotic methods, each of which gives only two particular solutions of (4.1). These methods will now be described and investigated mathematically in more detail, because Heisenberg's work has received some criticism in this connection.*

The first of these methods is to develop $\phi(y)$ in powers of $(\alpha R)^{-1}$. We put

$$
\phi(y)=\phi^{(0)}(y)+(\alpha R)^{-1} \phi^{(1)}(y)+(\alpha R)^{-2} \phi^{(2)}(y)+\cdots,
$$

and substitute in (4.1). Comparing corresponding powers of $(\alpha R)^{-1}$, we have the following differential equations

$$
\left.\begin{array}{r}
(w-c)\left(\phi^{(0) \prime \prime}-\alpha^{2} \phi^{(0)}\right)-w^{\prime \prime} \phi^{(0)}=0, \\
(w-c)\left(\phi^{(k) \prime \prime}-\alpha^{2} \phi^{(k)}\right)-w^{\prime \prime} \phi^{(k)}=-i\left[\phi^{(k-1) \text { iv }}-2 \alpha^{2} \phi^{(k-1) \prime \prime}+\alpha^{4} \phi^{(k-1)}\right], \\
(k \geqq 1) .
\end{array}\right\}
$$

The initial approximation satisfies the inviscid equation and can be solved by developing $\phi^{(0)}$ in powers of $\alpha^{2}$. Indeed, two particular integrals of (4.13) are

$$
\left.\begin{array}{l}
\phi_{1}^{(0)}=(w-c)\left[h_{0}(y)+\alpha^{2} h_{2}(y)+\alpha^{4} h_{4}(y)+\cdots\right], \\
\phi_{2}^{(0)}=(w-c)\left[k_{1}(y)+\alpha^{2} k_{3}(y)+\alpha^{4} k_{5}(y)+\cdots\right],
\end{array}\right\}
$$

where

$$
\left.\begin{array}{c}
h_{2 n+2}(y)=\int_{y_{1}}^{y} d y(w-c)^{-2} \int_{y_{1}}^{y} d y(w-c)^{2} h_{2 n}(y), \\
(n \geqq 0), \\
k_{1}(y)=\int_{y_{1}}^{y} d y(w-c)^{-2}, \quad k_{2 n+3}(y)=\int_{y_{1}}^{y} d y(w-c)^{2} \int_{y_{1}}^{y} d y(w-c)^{-2} k_{2 n+1}(y), \\
(n \geqq 0) .
\end{array}\right\}
$$

The point $y_{1}$ might have been any fixed point instead of one of the end points; but it is found convenient to take it this way.

Having found two particular integrals for $\phi^{(0)}$, we can obtain the higher approximations by quadratures. In actual calculations, this is not necessary

Because of the general nature of the Eq. (4.1), $\phi(y)$ is an entire function of $\alpha R$. Hence, the infinite point of the $\alpha R$-plane is a singular point, unless $\phi(y)$ is independent of $\alpha R$. Consequently, the series (4.12) is asymptotic, unless $\phi(y)$ is a polynomial in $(\alpha R)^{-1}$. We note also that (4.13) is of the second order, so that only two solutions are obtained by this method. The solutions of (4.13) are entire functions of $\alpha^{2}$ and hence the series (4.14) are uniformly convergent for any finite region of the complex $\alpha^{2}$-plane, for a fixed value of $y$, except when $y$ is the singular point $y_{0}$ of the differential equation (4.13).†

* Tollmien, loc. cit., 1929, p. 43.

† This can also be seen from the series itself. So long as it is possible to run a path of finite length from $y_{1}$ to $y$ on which $w-c \neq 0$, the general terms $\alpha^{2 n} h_{2 n}$ and $\alpha^{2 n+1} k_{2 n+1}$ of the two series are bounded by $A(\alpha M)^{2 n} /(2 n)$ ! and $B(\alpha M)^{2 n+1} /(2 n+1)$ !, respectively, $(A, B, M$ being (suitably) fixed constants), and 
In fact, the differential equation (4.13) has a logarithmic singularity at the point $y_{0}$. This point is, however, an ordinary point in the exact equation (4.1), and the singularity is introduced purely by the method of asymptotic integration. However, the appearance of this singularity gives a serious ambiguity in the determination of the correct path leading from $y_{1}$ to $y$ in order that (4.14) may give valid approximations to integrals of (4.1) all along the path.* The proper way to settle this question is to compare the solutions (4.14) with the asymptotic expansions of the regular solutions obtained by the previous method. This will be done later after we have described the second asymptotic method of Heisenberg for the other two particular integrals; for the same kind of problem also arises there.

To obtain two other integrals of (4.1) in asymptotic forms, let us make the transformation

$$
\phi=\exp \left\{\int g d y\right\}
$$

Then, we obtain the non-linear differential equation

$$
\begin{aligned}
& (w-c)\left\{\left(g^{2}+g^{\prime}\right)-\alpha^{2}\right\}-w^{\prime \prime} \\
& \quad=-\frac{i}{\alpha R}\left\{g^{4}+6 g^{2} g^{\prime}+3 g^{\prime 2}+4 g g^{\prime \prime}+g^{\prime \prime \prime}-2 \alpha^{2}\left(g^{2}+g^{\prime}\right)+\alpha^{4}\right\}
\end{aligned}
$$

for the function $g(y)$. We try to solve this by putting

$$
g(y)=(\alpha R)^{1 / 2} g_{0}(y)+g_{1}(y)+(\alpha R)^{-1 / 2} g_{2}(y)+\cdots .
$$

Then, we obtain the set of equations

$$
\begin{aligned}
& (w-c) g_{0}^{2}=-i g_{0}^{\prime}, \quad(w-c)\left(g_{0}^{\prime}+2 g_{0} g_{1}\right)=-i\left(4 g_{0}^{3} g_{1}+6 g_{0}^{2} g_{0}^{\prime}\right), \\
& (w-c)\left(g_{1}^{\prime}+g_{1}^{2}+2 g_{0} g_{2}-\alpha^{2}\right)-w^{\prime \prime}=-i\left(4 g_{0}^{3} g_{2}+6 g_{1}^{2}+2 g_{0} g_{1} g_{0}^{\prime}+3 g_{0}^{\prime 2}-2 \alpha^{2} g_{0}^{2}\right),
\end{aligned}
$$

Hence we can obtain the successive approximations without integration. Thus,

$$
g_{0}= \pm \sqrt{i(w-c)}, \quad g_{1}=-\frac{5}{2} \frac{g_{0}^{\prime}}{g_{0}}, \ldots .
$$

For definiteness, we define

hence the series converge like the cosine and the sine series, respectively. Heisenberg did not prove the convergence of these series, but stated that their convergence can be hoped to be sufficiently rapid for $\alpha^{2}$ of the order of unity (loc. cit., 1924, pp. 584, 587). This was made a point of criticism by Tollmien (loc. cit., 1929 , p. 43 ).

* Considerable dispute has arisen in this connection. Note that it is impossible to dispense with this difficulty by remarking that the two different determinations will differ only by a constant multiple of a particular integral. If we draw two paths from $y_{1}$ to $y$ and obtain such a difference in the solution, it is evident that the asymptotic solution cannot be valid on both paths, because the exact equation (4.1) has no singular point at $y=y_{0}$ and hence its solution must be single-valued. Although a mistake here would not cause serious difficulties so far as the numerical evaluation of the eigen-value problem is concerned, it does lead to misunderstanding and confusion elsewhere. Even after Heisenberg and Tollmien have analyzed this problem in some detail, they still take the very misleading step of taking the complex conjugate of the inviscid equation (Heisenberg, loc. cit., 1924, p. 596; Tollmien, loc. cit., 1935, p. 88). This point will be discussed more fully later. 


$$
\arg i=\frac{\pi}{2}, \quad \arg (w-c)>0 \text { for } w-c>0 .
$$

For negative values of $w-c$, we cannot decide, without further investigation, whether $\arg (w-c)=+\pi$ or $\arg (w-c)=-\pi$. The point $y_{0}$, where $w=c$, appeared in the previous asymptotic solution as a logarithmic branch point; here it is an algebraic branch point. The determination of the correct path should follow the same criterion as the other two integrals, that (4.18) gives two asymptotic solutions of the exact equation (4.1) all along the path. This path might be expected to be the same as that in the previous case. All these will be discussed in the next section.

After such a question is settled, substitution of (4.19) into (4.16) and (4.17) gives the two asymptotic solutions

$$
\left.\begin{array}{l}
\phi_{3}(y)=(w-c)^{-5 / 4} \exp \left\{-\int_{y_{0}}^{y} \sqrt{i \alpha R(w-c)} d y\right\}, \\
\phi_{4}(y)=(w-c)^{-5 / 4} \exp \left\{+\int_{y_{0}}^{y} \sqrt{i \alpha R(w-c)} d y\right\},
\end{array}\right\}
$$

where factors of the order $\exp (\alpha R)^{-1 / 2}=1+O\left\{(\alpha R)^{-1 / 2}\right\}$ are taken as unity.

5. Analytical properties of the solutions. Having thus obtained four asymptotic solutions of the equation (4.1), we must try to correlate them with the four solutions (4.9) and (4.11), and above all to study the correct determination of path around the artificial singularity introduced by the asymptotic methods. For this purpose, we consider the asymptotic expansions of the four regular solutions obtained by the first method and transfer back to the independent variable $y$.

Let us recall that the asymptotic cxpansions of the Hankel functions $H_{1 / 3}^{(1)}(\xi)$, $H_{1 / 3}^{(2)}(\xi)$ are given by [76],

$$
\begin{aligned}
& H_{1 / 3}^{(1)}(\xi) \sim\left(\frac{2}{\pi \xi}\right)^{1 / 2} \exp \left\{i\left(\xi-\frac{5 \pi}{12}\right)\right\}\left\{1+\sum_{r=1}^{\infty} \frac{(-)^{r}(1 / 3, r)}{(2 i \xi)^{r}}\right\}, \\
& (-\pi<\arg \xi<2 \pi), \\
& H_{1 / 3}^{(2)}(\xi) \sim\left(\frac{2}{\pi \xi}\right)^{1 / 2} \exp \left\{-i\left(-\frac{\pi}{12}\right)\right\}\left\{1+\sum_{r=1}^{\infty} \frac{(1 / 3, r)}{(2 i \xi)^{r}}\right\}, \\
& (-2 \pi<\arg \xi<\pi) .
\end{aligned}
$$

If we put $3 \xi=2\left(i \alpha_{0} \eta\right)^{3 / 2}$, then $(5.1)$ becomes

$$
\begin{array}{r}
H_{1 / 3}^{(1)}\left[\frac{2}{3}\left(i \alpha_{0} \eta\right)^{3 / 2}\right] \sim\left(\frac{3}{\pi}\right)^{1 / 2}\left(i \alpha_{0} \eta\right)^{-3 / 4} \exp \left\{\frac{2}{3}\left(\alpha_{0} \eta\right)^{3 / 2} e^{5 \pi i / 4}-\frac{5 \pi}{12}\right\}\left\{1+O\left(\eta^{-3 / 2}\right)\right\} \\
\left(-\frac{7 \pi}{6}<\arg \left(\alpha_{0} \eta\right)<\frac{5 \pi}{6}\right), \\
H_{1 / 3}^{(2)}\left[\frac{2}{3}\left(i \alpha_{0} \eta\right)^{3 / 2}\right] \sim\left(\frac{3}{\pi}\right)^{1 / 2}\left(i \alpha_{0} \eta\right)^{-3 / 4} \exp \left\{\frac{2}{3}\left(\alpha_{0} \eta\right)^{3 / 2} e^{\pi i / 4}+\frac{5 \pi}{12}\right\}\left\{1+O\left(\eta^{-3 / 2}\right)\right\} \\
\\
\left(-\frac{11 \pi}{6}<\arg \left(\alpha_{0} \eta\right)<\frac{\pi}{6}\right)
\end{array}
$$


With the help of these formulae and using the legitimate process of integrating the asymptotic expansions term by term, we obtain

$$
\begin{aligned}
\chi_{1}^{(0)}+\epsilon \chi_{1}^{(1)} & \sim \eta+\frac{\epsilon w_{0}^{\prime \prime}}{2 w_{0}^{\prime}} \eta^{2}=\frac{1}{w_{0}^{\prime} \epsilon}\left(w_{0}^{\prime} y+\frac{w_{0}^{\prime \prime}}{2} y^{2}\right), \\
\chi_{2}^{(0)}+\epsilon \chi_{2}^{(1)} & \sim 1+\epsilon \frac{w_{0}^{\prime \prime}}{w_{0}^{\prime}} \eta \log \eta \sim 1+\frac{w_{0}^{\prime \prime}}{w_{0}^{\prime}} y \log y, \\
\chi_{3}^{(0)} & \sim \text { const. } \eta^{-5 / 4} \exp \left\{\frac{2}{3}\left(\alpha_{0} \eta\right)^{3 / 2} e^{5 x i / 4}\right\} \\
& =\text { const. }\left(y-y_{0}\right)^{-5 / 4} \exp \left\{-\int_{y=1}^{y} \sqrt{i \alpha R w_{0}^{\prime}\left(y-y_{0}\right)} d y\right\}, \\
\chi_{4}^{(0)} & \sim \text { const. } \eta^{-5 / 4} \exp \left\{\frac{2}{3}\left(\alpha_{0} \eta\right)^{3 / 2} e^{x i / 4}\right\} \\
& =\text { const. }\left(y-y_{0}\right)^{-5 / 4} \exp \left\{\int_{y_{0}}^{y} \sqrt{i \alpha R w_{0}^{\prime}\left(y-y_{0}\right)} d y\right\} .
\end{aligned}
$$

These formulae can be easily seen to agree with the four asymptotic solutions (4.13) and (4.21) to the proper order of approximation, if we replace $y_{1}$ by $y_{0}$ in $\phi_{1}^{(0)}$ (which is permissible).

In evaluating the asymptotic expressions (5.3), the argument $\alpha_{0} \eta$ must satisfy both requirements specified in $(5.2)$, i.e.,

$$
-7 \pi / 6<\arg \left(\alpha_{0} \eta\right)<\pi / 6 .
$$

In this range, the asymptotic solutions (4.13) and (4.21) hold. Having thus established the range of validity of these solutions, we no longer need to make further comparisons of the two methods of solution.

At least three plans are now possible for further numerical work. First, we may use the four solutions obtained in the approximate form (4.9). Secondly, we may use the four asymptotic solutions (4.14) and (4.21). Thirdly, we may approximate $\left\{\phi_{1}, \phi_{2}, \phi_{3}, \phi_{4}\right\}$ by the four functions $\left\{\phi_{1}^{(0)}, \phi_{2}^{(0)}, \chi_{3}^{(0)}, \chi_{4}^{(0)}\right\}$ given by (4.14) and (4.9). The first method is very similar to the method used by Hopf [16] and Tietjens [72] for linear velocity distributions, where the exact solutions are given by functions of the general nature of those in (4.9). For curved velocity distributions, the functions $\chi_{1}^{(0)}, \chi_{2}^{(0)}$ do not give $\phi_{1}$ and $\phi_{2}$ with sufficient accuracy, and this plan is not good. The second plan was used by Heisenberg in his investigation of the stability of the Poiseuile flow; but he also realized that it served only part of his purpose, and he stated that the third plan should be used.* Tollmien substantially adopted the third plan for his investigation of the stability of the boundary layer, although he did not point out the connection of his method with Heisenberg's work. Instead of the expressions (4.14) for $\phi_{1}$ and $\phi_{2}$, he used solutions in the forms of power series in $y$. These solutions are easily manageable only for linear and parabolic velocity distributions. Accordingly, he tried to approximate the Blasius profile with such profiles. Since such approximations are not good enough in the neighborhood of the point $y=y_{0}$, where $w=c$, his discussion becomes very complicated. In the present work, we base our cal-

\footnotetext{
* Loc. cit., p. 404.
} 
culations upon the use of (4.14). It will be seen that our method can be applied to any profile with good accuracy. A comparison with Tollmien's method will be discussed in the Appendix to Part III.

It may be added that the adoption of the third plan leaves an error of the order of $(\alpha R)^{-1}$ in $\phi_{1}$ and $\phi_{2}$, and an error of the order of $(\alpha R)^{-1 / 3}$ in $\phi_{3}$ and $\phi_{4}$. These errors can be reduced by including the higher approximations. In practice, this is hardly necessary. A detailed discussion of numerical accuracy will be found in the Appendix to Part III.

Having thus established the region of validity of the asymptotic solutions, we shall try to settle a few questions of considerable dispute, namely, (a) the "crossing substitution," (b) the inner friction layers, and (c) the complex conjugate of the inviscid solution.

a) The crossing substitution. From previous discussions, it is evident that if we pass from $y>\operatorname{Re}\left(y_{0}\right)$ to $y<\operatorname{Re}\left(y_{0}\right)$ along a path below the point $y_{0}$, we are always in a region of the $y$-plane where the above asymptotic solutions hold, and no further investigation is necessary. In fact, if $c_{i}>0$ (and is small) and $\operatorname{Re}\left(w_{0}^{\prime}\right)>0$, the point $y_{0}$ is above the real axis, and the asymptotic solutions are valid along the real axis of $y$. In the case of real $c$, the point $y_{0}$ is on the real axis, and there is one point on the real axis where the asymptotic solutions fail to be valid. In the case $c_{i}<0$, and $\operatorname{Re}\left(w_{0}^{\prime}\right)>0$, the point $y_{0}$ is below the real axis, and the lines arg $\left\{\alpha_{0}\left(y-y_{0}\right)\right\}=-7 \pi / 6, \pi / 6$ intersect the real axis in two points $y_{f}^{\prime}, y_{f}^{\prime \prime}$ with* $y_{1}<y_{f}^{\prime}<y_{f}^{\prime \prime}<y_{2}$. Thus, the asymptotic expressions (4.14) and (4.21) represent one solution for $y_{1} \leqq y<y_{f}^{\prime}$ and

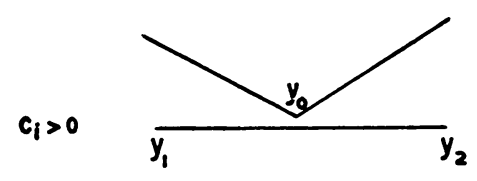
$y_{f}<y \leqq y_{2}$, but not the same solution for $y_{f}^{\prime}<y<y_{f}^{\prime \prime}$. It is necessary to obtain a suitable "crossing substitution" in order to obtain the correct solutions for $\pi / 6<\arg \left\{\alpha_{0}\left(y-y_{0}\right)\right\}<5 \pi / 6$ (i.e., in crossing the lines arg $\left.\left\{\alpha_{0}\left(y-y_{0}\right)\right\}=-7 \pi / 6, \pi / 6\right)$. For this purpose, we must obtain the asymptotic expansion of the Hankel functions $H_{1 / 3}^{(j)}\left[2\left(i \alpha_{0} \eta^{3 / 2}\right) / 3\right],(j=1,2)$, proper to that region. The analytical expression for $H_{1 / 3}^{(2)}$ would then be quite different from that given in (5.2). Thus, in crossing the two points $y_{f}^{\prime}$ and $y_{f}^{\prime \prime}$ of the real axis, the asymptotic solutions fail to be analytic. However, it is to be noted that the failure of the asymptotic solutions along the real axis does not exclude their use in the investigation of the boundary value problems to be considered below, so long as we are concerned only with the eigen-value problem. It is only necessary that these solutions be valid in a connected region containing the end-points $y_{1}$ and $y_{2}$. The calculation of the amplitude distribution of the disturbance (the eigen-function) in the neighborhood of the inner friction layers, however, is to be made with the regular solution, or we can calculate the eigen-function for $y_{f}^{\prime}<y<y_{f}^{\prime \prime}$ by using a proper

* The whole theory must be modified for extremely highly damped solutions for which $y_{f}^{\prime}<y_{1}<y_{2}<y_{f}^{\prime \prime}$. 
"crossing substitution." Since we are chiefly concerned with the eigen-value problem, we shall not go into further details.

In order to make the situation still clearer, let us see what would happen if we try to obtain our solutions for $y_{f}^{\prime}<y<y_{f}^{\prime \prime}$ by going along a path above the point $y_{0}$. For simplicity, let us take the case of real $c$ with $w_{0}^{\prime}>0$, and consider the asymptotic expressions $\phi_{3}^{(0)}$ and $\phi_{4}^{(0)}$ given by (5.3). We have ( $A, B$ being arbitrary constants)

$$
\begin{array}{lll}
\phi_{3}^{(0)} \sim A \eta^{-5 / 4} \exp \left\{\frac{2}{3}\left(\alpha_{0} \eta\right)^{3 / 2} e^{5 \pi i / 4}\right\}, & & (\eta>0), \\
\phi_{3}^{(0)} \sim A|\eta|^{-5 / 4} \exp \left\{\frac{2}{3}\left(\alpha_{0}|\eta|\right)^{3 / 2} e^{-\pi i / 4}+5 \pi i / 4\right\}, & & (\eta<0) ; \\
\phi_{4}^{(0)} \sim B \eta^{-5 / 4} \exp \left\{\frac{2}{3}\left(\alpha_{0} \eta\right)^{3 / 2} e^{\pi i / 4}\right\}, & & (\eta>0), \\
\phi_{4}^{(0)} \sim B|\eta|^{-5 / 4} \exp \left\{\frac{2}{3}\left(\alpha_{0}|\eta|\right)^{3 / 2} e^{-5 \pi i / 4}+5 \pi i / 4\right\}, & (\eta<0) .
\end{array}
$$

These are obtained by taking a path below the point $y_{0}$. If we had taken the other path, then $\arg (\eta)=\pi$ for $\eta<0$, and we would have the functions $\widetilde{\phi}_{3}^{(0)}$ and $\tilde{\phi}_{4}^{(0)}$, which agree with $\phi_{3}$ and $\phi_{4}$ for $\eta>0$, but are defined by

$$
\begin{aligned}
& \tilde{\phi}_{3}^{(0)} \sim A|\eta|^{-5 / 4} \exp \left\{\frac{2}{3}\left(\alpha_{0}|\eta|\right)^{3 / 2} e^{3 \pi i / 4}-5 \pi i / 4\right\}, \\
& \tilde{\phi}_{4}^{(0)} \sim B|\eta|^{-5 / 4} \exp \left\{\frac{2}{3}\left(\alpha_{0}|\eta|\right)^{3 / 2} e^{7 \pi i / 4}-5 \pi i / 4\right\},
\end{aligned}
$$

for $\eta<0$. Thus, if $A$ and $B$ are taken to be the same, we have

$$
\tilde{\phi}_{3}^{(0)}=-i \phi_{4}^{(0)}, \quad \tilde{\phi}_{4}^{(0)}=-i \phi_{3}^{(0)}, \quad \text { for } \eta<0 .
$$

Hence if we took $\tilde{\phi}_{3}^{(0)}$ and $\tilde{\phi}_{4}^{(0)}$ as the proper determinations, we would have to make the following "crossing substitution" corresponding to a passage from $\eta>0$ to $\eta<0$ : $\tilde{\phi}_{3}^{(0)} \rightarrow \tilde{\phi}_{4}^{(0)} ; \widetilde{\phi}_{4}^{(0)} \rightarrow i \widetilde{\phi}_{3}^{(0)}$. If we note that $\widetilde{\phi}_{3}^{(0)} \ll \tilde{\phi}_{4}^{(0)}$ both for $w-c>0$ and for $w-c<0$, we would also have the following equivalent change: $\tilde{\phi}_{3}^{(0)} \rightarrow \widetilde{\phi}^{(0)}+i \widetilde{\phi}_{4}^{(0)} ; \widetilde{\phi}_{3}^{(0)}-i \widetilde{\phi}_{4}^{(0)} \rightarrow \widetilde{\phi}_{3}^{(0)}$. These may be compared with Eq. (16), p. 589 of Heisenberg's paper. In making the comparison, we note his definition of the angle of $w-c$ (p. 585), and the difference of notation in the fundamental equation of stability.

The first study of "the crossing substitution" seems to be due to Stokes in connection with the asymptotic expansions of Bessel functions. It may therefore be properly designated as Stokes' phenomenon [76]. We should also compare our results with the work of Jeffreys [17], the $W-K-B$ method [23] in quantum mechanics, ${ }^{*}$ and the mathematical investigations of Langer [24] and others. ${ }^{* *}$ In those cases, a differential equation of the form $\epsilon^{3} \phi^{\prime \prime}+q(y) \phi=0$ is considered. If this equation is treated by the method of $\S 4$ by writing $\phi=\chi^{(0)}(\eta)+\epsilon \chi^{(1)}(\eta)+\cdots, y-y_{0}=\epsilon \eta$, and $q(y)=q_{0}^{\prime}(\epsilon \eta)+\frac{1}{2} q_{0}^{\prime \prime}(\epsilon \eta)^{2}+\cdots$, the equation for $\chi^{(0)}(\eta)$ is $\chi^{(0) \prime \prime}+q_{0}^{\prime} \eta \chi^{(0)}=0$ as compared with (4.7), $\chi^{(0) \text { iv }}-i \eta w_{0}^{\prime} \chi^{(0) \prime \prime}=0$. It is evident that our $\eta$ corresponds to in in their case. Kramers has shown that the cuts in their asymptotic expansions are the lines $\arg (\eta)= \pm \pi / 3$. Thus, in our case, the cuts should be arg $(\eta)=\pi / 6,5 \pi / 6$. This agrees with our previous discussions. An important difference is the following. In

* I am indebted to Professor P. S. Epstein for calling my attention to this comparison.

** For example, S. Goldstein, Proc. Lond. Math. Soc. (2) 28, 81-90 (1928); C. C. Hurd, Tôhoku Math. Journ. 45, 58-68 (1939), and the papers of W. J. Trjitzinsky and others quoted there. 
their case, the two boundary points on the real axis are separated into two regions of the complex plane by the cuts, so that a crossing substitution is absolutely necessary. In our case, the two boundary points on the real axis belong to the same region, and a crossing substitution is superfluous, so far as the eigen-value problem is concerned.

b) The inner friction layers. There is also a very significant physical interpretation associated with the "crossing substitution" of the asymptotic solutions. The initial approximations $\phi_{1}^{(0)}$ and $\phi_{2}^{(0)}$ satisfy the inviscid equation. Hence, if $c_{i}>0$, these solutions hold throughout the interval $\left(y_{1}, y_{2}\right)$ of the real axis, and the effect of viscosity is entirely negligible inside the fluid for sufficiently large Reynolds numbers. If $c_{i} \leqq 0$, the inviscid solution can never hold all along the real axis, and hence the effect of viscosity inside the fluid is not negligible, however large the Reynolds number may be. The singularity of the asymptotic solutions means a very rapid change of velocity within a small distance so that the effect of viscosity is no longer negligible there. Physically, such a point on the real axis corresponds to a layer of fluid where the viscous forces play an important role.

Referring to the foregoing discussions, we see that there are two inner friction layers for the damped oscillations, one for the neutral oscillations, and none for the self-excited oscillations.

In the neutral case, the first term of (4.1) disappears at the critical layer $w=c$. The equation then represents a balancing of the vorticity transferred by the disturbance and that diffused away by the effect of viscosity. It is therefore understandable that the effect of viscosity must be predominant there. In the other two cases, $w-c$ never vanishes in the fluid, there is the vorticity carried by the main flow (relative to an observer moving with the phase velocity $c_{r}$ ) and there is always the change of vorticity due to amplification or damping. In the case of amplified oscillations, these two effects can be in equilibrium with the transfer of vorticity due to the disturbance, and the effect of viscosity is completely negligible at very large Reynolds numbers. In the case of damped oscillations, these effects presumably never balance each other, thus resulting in the formation of two critical layers, where the effect of viscosity is not negligible.

c) The complex conjugate of the solution $\phi(y)$. It is often argued,* that if $\phi(y)$ is a solution of the inviscid equation with an eigen-value $c$, then $\phi(y)$ is another solution with the eigen-value $\bar{c}$, satisfying the same real boundary conditions on the real axis. Thus, to each damped solution, there is always a corresponding amplified solution, and vice versa. This argument is in direct contradiction to the foregoing discussions, because an amplified solution and a damped solution have entirely different characteristics with respect to inner friction layers. It appears, therefore, that $\phi(y)$ should still represent a solution of the same nature as $\phi(y)$.

This is indeed the case, and can be seen more clearly from an examination of the complete equation (4.1). If we take the complex conjugate of that equation, and write $y$ for $\bar{y}$ (which is essentially done in the usual argument), we have

$$
\{w(y)-c\}\left\{\Phi^{\prime \prime}-\alpha^{2} \bar{\phi}\right\}-w^{\prime \prime}(y) \Phi=\frac{i}{\alpha R}\left\{\Phi^{\mathrm{iv}}-2 \alpha^{2} \phi^{\prime \prime}+\alpha^{4} \phi\right\} .
$$

${ }^{*}$ Heisenberg, loc. cit. p. 596; Tollmien, loc. cit., 1935, p. 88. The failure of such an argument would indicate that Heisenberg's classification of velocity profiles on p. 597 of his paper is untenable. 
The complete stream function $\Psi^{\prime}(x, y, t)$ satisfying this equation is

$$
\psi^{\prime}(x, y, t)=\Phi(y) e^{-i \alpha(x-\bar{c} t)} .
$$

Thus, if $\operatorname{Im}(c)<0$, then $\operatorname{Im}(\bar{c})>0$, and we still have a damped solution. This should also hold for the inviscid equation, since it is regarded as a limiting case of the viscous equation. From the inviscid equation itself, there is no way of telling whether $\operatorname{Im}(\bar{c})>0$ corresponds to damping or to amplification. In fact, the asymptotic solutions of Eq. (5.5) (including the limiting inviscid solutions) hold for

$$
-\pi / 6<\arg \left\{\bar{\alpha}_{0}\left(y-y_{0}\right)\right\}<7 \pi / 6, \quad w\left(y_{0}\right)=\bar{c} .
$$

Thus, we have a solution $\phi(y)$, valid in a region which is quite improper for an asymptotic solution of (4.1). [Compare (5.4) and (5.6).] Hence, it is not legitimate to conclude that a solution of a different nature can be obtained by taking the complex conjugate. The influence of these discussions upon the usual conclusions regarding stability in an inviscid fluid will be discussed fully in the next part of the paper.

6. The boundary value problems. Having fully investigated the solutions of the equation of disturbance, we shall now turn to a study of the boundary-value problems which have been taken up briefly at the end of $\$ 3$. In general, the boundary conditions are essentially that the velocities of the disturbance should vanish on the solid boundaries, and also at infinity if the field of flow extends to infinity. However, it is often convenient to use equivalent boundary conditions for certain types of velocity distributions.

In order not to be lost in too much generality, we shall limit ourselves to three classes of velocity distributions (as specified below and shown in Fig. 3), and select our fundamental interval $\left(y_{1}, y_{2}\right)$ so that $w^{\prime}(y) \geqq 0$ for $y_{1} \leqq y \leqq y_{2}$. We shall define our characteristic length so that $y_{2}-y_{1}=1$, and let $\phi_{1}(y ; c, \alpha, \alpha R), \phi_{2}(y ; c, \alpha, \alpha R), \phi_{3}(y ; c, \alpha, \alpha R)$, $\phi_{4}(y ; c, \alpha, \alpha R)$ be a fundamental system of solutions (4.1) arranged in the order discussed above.

CASE (1). Flow between solid walls in relative

(1)

(2)

(3)

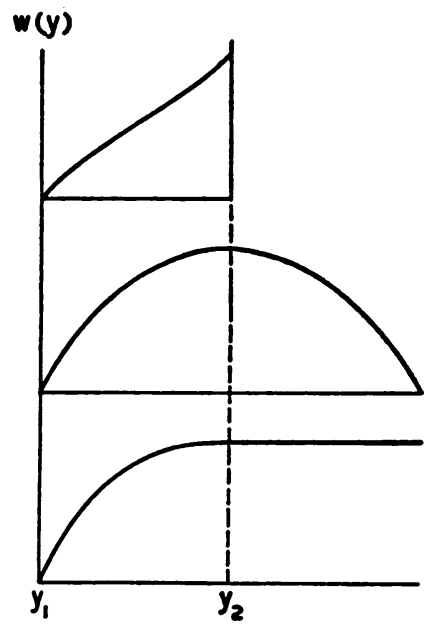

Fig. 3. The three types of velocity distributions. motion. In this case, the boundary conditions are given by

$$
\phi\left(y_{1}\right)=\phi^{\prime}\left(y_{1}\right)=\phi\left(y_{2}\right)=\phi^{\prime}\left(y_{2}\right)=0,
$$

because the velocity of the disturbance should vanish on both the solid boundaries. The determinantal equation corresponding to these conditions is

$$
F_{1}(\alpha, c, \alpha R)=\left|\begin{array}{llll}
\phi_{11} & \phi_{21} & \phi_{31} & \phi_{41} \\
\phi_{12} & \phi_{22} & \phi_{32} & \phi_{42} \\
\phi_{11}^{\prime} & \phi_{21}^{\prime} & \phi_{31}^{\prime} & \phi_{41}^{\prime} \\
\phi_{12}^{\prime} & \phi_{22}^{\prime} & \phi_{32}^{\prime} & \phi_{42}^{\prime}
\end{array}\right|=0,
$$

where $\phi_{11}, \phi_{11}^{\prime}$, etc., stand for $\phi_{1}\left(y_{1}\right), \phi_{1}^{\prime}\left(y_{1}\right)$, etc. In this and all later discussions, a 
subscript 1 or 2 attached to a function of $y$ shall denote the value of that function at $y=y_{1}$ or $y=y_{2}$ respectively.

CASE (2). Symmetrical flow between solid walls at rest. In this case, it is easily seen from (4.1) that the disturbance can be separated into two independent parts, one symmetrical with respect to the line $y=y_{2}$ and the other antisymmetrical. (a) If $\phi(y)$ is a symmetrical function (antisymmetrical disturbance), then the conditions

$$
\phi\left(y_{1}\right)=\phi^{\prime}\left(y_{1}\right)=\phi^{\prime}\left(y_{2}\right)=\phi^{\prime \prime \prime}\left(y_{2}\right)=0
$$

hold, and we have the determinantal equation

$$
F_{2}(\alpha, c, \alpha R)=\left|\begin{array}{cccc}
\phi_{11} & \phi_{21} & \phi_{31} & \phi_{41} \\
\phi_{11}^{\prime} & \phi_{21}^{\prime} & \phi_{31}^{\prime} & \phi_{41}^{\prime} \\
\phi_{12}^{\prime} & \phi_{22}^{\prime} & \phi_{32}^{\prime} & \phi_{42}^{\prime} \\
\phi_{12}^{\prime \prime \prime} & \phi_{22}^{\prime \prime \prime} & \phi_{32}^{\prime \prime \prime} & \phi_{42}^{\prime \prime \prime}
\end{array}\right|=0 .
$$

(b) If $\phi(y)$ is an odd function of $y-y_{2}$ (symmetrical disturbance), then the boundary conditions are

$$
\phi\left(y_{1}\right)=\phi^{\prime}\left(y_{1}\right)=\phi\left(y_{2}\right)=\phi^{\prime \prime}\left(y_{2}\right)=0,
$$

and we have the relation

$$
F_{3}(\alpha, c, \alpha R)=\left|\begin{array}{llll}
\phi_{11} & \phi_{21} & \phi_{31} & \phi_{41} \\
\phi_{12} & \phi_{22} & \phi_{32} & \phi_{42} \\
\phi_{11}^{\prime} & \phi_{21}^{\prime} & \phi_{31}^{\prime} & \phi_{41}^{\prime} \\
\phi_{12}^{\prime \prime} & \phi_{22}^{\prime \prime} & \phi_{32}^{\prime \prime} & \phi_{42}^{\prime \prime}
\end{array}\right|=0 .
$$

CASE (3). Flow of the boundary-layer type. In this case, the point $y_{2}$ is taken to correspond to the "edge" of the boundary layer, beyond which the velocity is substantially constant. The boundary conditions to be satisfied at $y_{1}$ are the usual ones;

$$
\phi\left(y_{1}\right)=\phi^{\prime}\left(y_{1}\right)=0 .
$$

The boundary conditions for $y$ becoming infinite are to be replaced as follows. ${ }^{*}$ Since the particular integral $\phi_{4}$ becomes infinite as $y$ becomes infinite, our boundary condition requires that $\phi$ is a linear combination of $\phi_{1}, \phi_{2}, \phi_{3}$ alone. Thus,

$$
\phi=C_{1} \phi_{1}+C_{2} \phi_{2}+C_{3} \phi_{3},
$$

where $C_{1}, C_{2}, C_{3}$ are constants of integration. Also, the integral $\phi_{3}$ makes practically no contribution for $y \geqq y_{2}$ so that we expect $\phi(y)$ to satisfy the inviscid equation for $y \geqq y_{2}$. Here $w^{\prime \prime}=0$, and hence two particular integrals are $e^{ \pm \alpha y}$. The condition that $\phi \rightarrow 0$ as $y \rightarrow \infty$ excludes the integral $e^{\alpha \nu}$. Hence, $\phi$ must be proportional to $e^{-\alpha y}$ for $y>y_{2}$. This may be expressed as follows:

$$
\phi^{\prime}+\alpha \phi=0 \text { for } y \geqq y_{2} .
$$

Hence, we have the determinantal equation

* Cf. Tietjens [72] and Tollmien [73]. 


$$
F_{1}(\alpha, c, \alpha R)=\left|\begin{array}{lll}
\phi_{11} & \phi_{21} & \phi_{31} \\
\phi_{12}^{\prime}+\alpha \phi_{12} & \phi_{22}^{\prime}+\phi \alpha_{22} & 0 \\
\phi_{11}^{\prime} & \phi_{21}^{\prime} & \phi_{31}^{\prime}
\end{array}\right|=0
$$

We note that the point $y_{2}$ can be replaced by any value of $y>y_{2}$. This is equivalent to the fact that the thickness of the boundary layer cannot be definitely defined. The larger this thickness is taken, the more accurate the results should be .*

The functions $F_{1}, F_{2}, F_{3}$, and $F_{4}$ are entire functions of the parameters $\alpha, c$, and $R$.

Reduction of the equations for large values of $\alpha R$. The equations (6.2), (6.4), and (6.6) can be substantially simplified for large values of $\alpha R$. Referring to (4.21), we see that $\phi_{3}(y)=A(y) e^{-Y}, \phi_{4}(y)=B(y) e^{Y}$, where $A(y)$ and $B(y)$ are of the order of unity, and $Y$ is defined by the relation

$$
Y=\int_{y_{1}}^{y} \sqrt{i \alpha R(w-c)} d y .
$$

Hence, we have the following relations, giving the order of magnitude of certain quantities :

$$
\begin{aligned}
& \frac{\phi_{31}^{\prime}}{\phi_{31}}=-\sqrt{i \alpha R\left(w_{1}-c\right)}+\frac{A_{1}^{\prime}}{A_{1}}, \quad \frac{\phi_{32}}{\phi_{31}}=\frac{A_{2}}{A_{1}} e^{-P}, \\
& \frac{\phi_{32}^{\prime}}{\phi_{31}}=\left\{-\sqrt{i \alpha R\left(w_{2}-c\right)} \frac{A_{2}}{A_{1}}+\frac{A_{2}^{\prime}}{A_{1}}\right\} e^{-P}, \\
& \frac{\phi_{32}^{\prime \prime}}{\phi_{31}}=\left\{i \alpha R\left(w_{2}-c\right) \frac{A_{2}}{A_{1}}+O(\sqrt{\alpha R)}\} e^{-P},\right. \\
& \frac{\phi_{32}^{\prime \prime \prime}}{\phi_{31}}=\left\{-\left[i \alpha R\left(w_{2}-c\right)\right]^{3 / 2} \frac{A_{2}}{A_{1}}+O(\alpha R)\right\} e^{-P} ; \\
& \frac{\phi_{41}^{\prime}}{\phi_{41}}=\sqrt{i \alpha R\left(w_{1}-c\right)}+\frac{B_{1}^{\prime}}{B_{1}}, \quad \frac{\phi_{42}}{\phi_{41}}=\frac{B_{2}}{B_{1}} e^{P}, \\
& \frac{\phi_{42}^{\prime}}{\phi_{41}}=\left\{\sqrt{i \alpha R\left(w_{2}-c\right)} \frac{B_{2}}{B_{1}}+\frac{B_{2}^{\prime}}{B_{1}}\right\} e^{P}, \\
& \frac{\phi_{42}^{\prime \prime}}{\phi_{41}}=\left\{i \alpha R\left(w_{2}-c\right) \frac{B_{2}}{B_{1}}+O(\sqrt{\alpha R)}\} e^{P},\right. \\
& \frac{\phi_{42}^{\prime \prime \prime}}{\phi_{41}^{\prime \prime}}=\left\{\left[i \alpha R\left(w_{2}-c\right)\right]^{3 / 2} \frac{B_{2}}{B_{1}}+O(\alpha R)\right\} e^{P},
\end{aligned}
$$

where

$$
P=\int_{y_{1}}^{y_{2}} \sqrt{i \alpha R(w-c)} d y .
$$

It then appears that the sign of the real part of $P$ is of consequence. It can be veri-

* In later calculation of the Blasius case, we shall take a boundary layer about 1.19 times as thick as that used by Tollmien. 
fied that it is always positive when $c_{i}>0$. For then the path of integration can be taken along the real axis of $y$, and we have $-\pi<\arg (w-c)<0$; consequently, $\dot{-} \pi / 4<\arg (P)<\pi / 4$. With reference to $(4.21),(6.11)$ and $(6.12)$, we see that the condition $P=n \pi i$ ( $n=$ an integer), expresses the fact that $\phi_{31}{ }^{\prime} \phi_{42}{ }^{\prime}=\phi_{41}{ }^{\prime} \phi_{32}{ }^{\prime}$ when terms of the order $(\alpha R)^{1 / 2}$ are neglected. This is the corrected form of the first solution of Heisenberg as expressed by the condition (27) on p. 596 of his paper. Heisenberg also stated that such a condition can only be satisfied for damped solutions. In fact, from the condition just obtained for $c_{i}>0$, we see that $\operatorname{Re}(P)$ can be negative only for highly damped solutions, for which the whole discussion must be modified. (Cf. footnote on p. $29, \S 5$.)

Neglecting quantities of the order $e^{-P}$ and $(\alpha R)^{-1}$ against quantities of the order of unity, we have the following simplifications of Eqs. (6.2), (6.4), and (6.6) for Cases (1) and (2).

CASE (1). Flow between solid walls in relative motion. We have

$$
\frac{f_{1}(\alpha, c)}{f_{3}(\alpha, c)}=\frac{\phi_{31}}{\phi_{31}^{\prime}}+\frac{\phi_{42}}{\phi_{42}^{\prime}} \frac{f_{2}(\alpha, c)}{f_{4}(\alpha, c)} .
$$

CASE (2a). Antisymmetrical disturbance in a symmetrical flow between solid walls. We have

$$
f_{2}(\alpha, c) / f_{4}(\alpha, c)=\phi_{31} / \phi_{31}^{\prime} .
$$

CASE (2b). Symmetrical disturbance in a symmetrical flow between solid walls. We have

$$
f_{1}(\alpha, c) / f_{3}(\alpha, c)=\phi_{31} / \phi_{31}^{\prime} \text {. }
$$

In these equations, the functions $f_{1}(\alpha, c), f_{2}(\alpha, c), f_{3}(\alpha, c)$ and $f_{4}(\alpha, c)$ are defined as follows :

$$
\begin{array}{ll}
f_{1}(\alpha, c)=\left|\begin{array}{ll}
\phi_{11} & \phi_{12} \\
\phi_{21} & \phi_{22}
\end{array}\right|, & f_{2}(\alpha, c)=\left|\begin{array}{ll}
\phi_{11} & \phi_{12}^{\prime} \\
\phi_{21} & \phi_{22}^{\prime}
\end{array}\right|, \\
f_{3}(\alpha, c)=\left|\begin{array}{ll}
\phi_{11}^{\prime} & \phi_{12} \\
\phi_{21}^{\prime} & \phi_{22}
\end{array}\right|, & f_{4}(\alpha, c)=\left|\begin{array}{ll}
\phi_{11}^{\prime} & \phi_{12}^{\prime} \\
\phi_{21}^{\prime} & \phi_{22}^{\prime}
\end{array}\right| .
\end{array}
$$

These functions depend only on $\alpha$ and $c$, because we may take the inviscid solutions for $\phi_{1}$ and $\phi_{2}$, which are accurate up to the order of $(\alpha R)^{-1}$. It may be reiterated that in computing $\phi_{12}, \phi_{22}, \phi_{12}^{\prime}, \phi_{22}^{\prime}$ in (6.16), we must take the path leading from $y_{1}$ to $y_{2}$ in the lower half plane.

CASE (3). Flow in the boundary layer along a flat plate. In this case we can reduce (6.10) to

$$
\frac{f_{2}+\alpha f_{1}}{f_{4}+\alpha f_{3}}=\frac{\phi_{31}}{\phi_{31}^{\prime}}
$$

if we also replace $\phi_{1}$ and $\phi_{2}$ by their inviscid approximations. The equations (6.13), (6.14), (6.15), and (6.17) are the final equations based on which the stability investigations are to be made.

The inviscid case. In the limit $\alpha R \rightarrow \infty$. The above equations reduce to 


$$
\begin{aligned}
f_{1}(\alpha, c)=0 & \text { for Case }(1) \text { and Case }(2 b) \\
f_{2}(\alpha, c)=0 & \text { for Case }(2 a) \\
f_{2}+\alpha f_{1}=0 & \text { for Case }(3)
\end{aligned}
$$

Mathematically, these are equivalent to the solution of the inviscid equation

$$
(w-c)\left(\phi^{\prime \prime}-\alpha^{2} \phi\right)-w^{\prime \prime} \phi=0
$$

subjected to one of the following three sets of boundary conditions

$\phi\left(y_{1}\right)=\phi\left(y_{2}\right)=0, \quad \phi\left(y_{1}\right)=\phi^{\prime}\left(y_{2}\right)=0, \quad \phi\left(y_{1}\right)=\phi^{\prime}\left(y_{2}\right)+\alpha \phi\left(y_{2}\right)=0$.

We have thus arrived at the conclusion that some asymptotic behavior of the stability conditions can be obtained by neglecting the effect of viscosity (provided proper care is given to the inner friction layer). This was tacitly assumed in the work of Rayleigh and others, while Heisenberg pointed out [loc. cit., p. 583] that a proof was necessary in accordance with some remarks of Oseen (38); he also virtually gave the proof.

In the next part of the paper, we shall therefore consider the simpler problem of an inviscid fluid. After a thorough investigation of that problem, we shall investigate the effect of viscosity by considering Eqs. (6.13), (6.14), (6.15), and (6.17) in greater detail. These results may be compared with the earlier ones of Heisenberg and Tollmien.

Numerical calculations of the stability limit based upon these equations will also be carried out for certain important special cases. For all these purposes, the evaluation of the six functions $f_{1}(\alpha, c), f_{2}(\alpha, c), f_{3}(\alpha, c), f_{4}(\alpha, c), \phi_{31} / \phi_{31}^{\prime}, \phi_{42} / \phi_{42}^{\prime}$ is necessary. We shall discuss this briefly here.

1) Evaluation of $f_{1}(\alpha, c), f_{2}(\alpha, c), f_{3}(\alpha, c), f_{4}(\alpha, c)$. These quantities are related to the inviscid solutions given by (4.14) with the path of integration subjected to the condition (5.4). Hence, we have

$$
\begin{aligned}
& \phi_{11}=-c, \quad \phi_{21}=0, \quad \phi_{11}^{\prime}=w_{1}^{\prime}, \quad \phi_{21}^{\prime}=-\frac{1}{c}, \\
& \phi_{12}=(1-c) \sum_{n=0}^{\infty} \alpha^{2 n} H_{2 n}(c), \quad \phi_{22}=(1-c) \sum_{n=0}^{\infty} \alpha^{2 n} K_{2 n+1}(c), \\
& \phi_{12}^{\prime}=(1-c)^{-1} \sum_{n=0}^{\infty} \alpha^{2 n} H_{2 n-1}(c)+(1-c)^{-1} w_{2}^{\prime} \phi_{12}, \\
& \phi_{22}^{\prime}=(1-c)^{-1} \sum_{n=0}^{\infty} \alpha^{2 n} K_{2 n}(c)+(1-c)^{-1} w_{2}^{\prime} \phi_{22},
\end{aligned}
$$

where

$$
\left.\begin{array}{rlrl}
H_{2 n}(c) & =h_{2 n}\left(y_{2}\right), & H_{2 n-1}(c) & =(1-c)^{-2} h_{2 n}^{\prime}\left(y_{2}\right), \\
K_{2 n+1}(c) & =k_{2 n+1}\left(y_{2}\right), & K_{2 n}(c) & =(1-c)^{2} k_{2 n+1}^{\prime}\left(y_{2}\right)
\end{array}\right\}
$$

are functions of $c$ alone. In the above evaluations, we have put $w_{1}=0$, in accordance 
with the actual conditions in all the cases considered. We have also chosen the characteristic velocity so that $w_{2}=1$. Referring to (6.23), we have

$$
\left.\begin{array}{ll}
f_{1}(\alpha, c)=-c \phi_{22}, & f_{2}(\alpha, c)=-c \phi_{22}^{\prime}, \\
f_{3}(\alpha, c)=w_{1}^{\prime} \phi_{22}+\frac{1}{c} \phi_{12}, & f_{4}(\alpha, c)=w_{1}^{\prime} \phi_{22}^{\prime}+\frac{1}{c} \phi_{12}^{\prime} .
\end{array}\right\}
$$

The actual evaluation therefore depends upon the calculation of the integrals (6.25).

2) Evaluation of $\phi_{31} / \phi_{31}^{\prime}, \phi_{42} / \phi_{42}^{\prime}$. These quantities are related to the highly oscillating integrals $\phi_{3}$ and $\phi_{4}$. For values of $\alpha R$ so large that both $(\alpha R)^{1 / 3} c,(\alpha R)^{1 / 3}(1-c)$ $\gg 1$, the approximate values of $\phi_{31} / \phi_{31}^{\prime}$ and $\phi_{42} / \phi_{42}^{\prime}$ are given by using (4.21). Thus,

$$
\frac{\phi_{31}}{\phi_{31}^{\prime}}=-\frac{e^{\pi i / 4}}{\sqrt{\alpha R c}}, \quad \frac{\phi_{42}}{\phi_{42}^{\prime}}=\frac{e^{-\pi i / 4}}{\sqrt{\alpha R(1-c)}} .
$$

The condition $(\alpha R)^{1 / 3}(1-c) \gg 1$ is generally satisfied, because $c$ is usually small and $\alpha R$ is usually very large. This is especially true for Eq. (6.13), for it will be seen from considerations of an inviscid fluid that this case is relatively stable. For the condition $(\alpha R)^{1 / 3} c \gg 1$, the situation is different, because $c$ is usually small. It is then more convenient to approximate $\phi_{3}$ by $\chi_{3}^{(0)}(\eta)$ given by (4.9) (or with higher approximations, if so desired). We then have

$$
\phi_{31} / \phi_{31}^{\prime}=\left(y_{1}-y_{0}\right) F(z),
$$

where

$$
F(z)=\frac{\int_{+\infty}^{-z} d \zeta \int_{+\infty}^{\zeta} \zeta^{1 / 2} d \zeta H_{1 / 3}^{(1)}\left[\frac{2}{3}(i \zeta)^{3 / 2}\right]}{-z \int_{+\infty}^{-z} d \zeta \zeta^{1 / 2} H_{1 / 3}^{(1)}\left[\frac{2}{3}(i \zeta)^{3 / 2}\right]}, \quad z=-\alpha_{0}\left(\eta_{1}-\eta_{0}\right) .
$$

This function has been calculated numerically by Tietjens for real values of $z$. We have made slightly more extensive and more accurate calculations. The present result differs slightly from that of Tietjens and is here tabulated in Table I and plotted in Fig. 4 together. with the related function $\mathcal{F}(z)$ defined by

$$
\mathcal{F}(z)=[1-F(z)]^{-1} .
$$

The asymptotic form of $F(z)$ is

$$
F(z) \sim z^{-3 / 2} e^{\pi i / 4}, \quad(z \gg 1) .
$$

This agrees with (6.27) if $\alpha_{0}^{3}\left(y_{1}-y_{0}\right)=w_{0}^{\prime}\left(y_{1}-y_{0}\right)$ is replaced by $-c$.

Acknowledgement. The author wishes to express his sincere gratitude to Professor Theodore von Kármán for suggesting this problem and for his invaluable help throughout this work; to Professor Clark B. Millikan for helpful suggestions and for the use of some of his unpublished notes, from which much inspiration and assistance have been derived; to Professors P. S. Epstein, H. Bateman, Dr. H. W. Liepmann and others for many valuable discussions and suggestions. The author is indebted to Mr. L. Lees for helpful criticism of the final manuscript. 


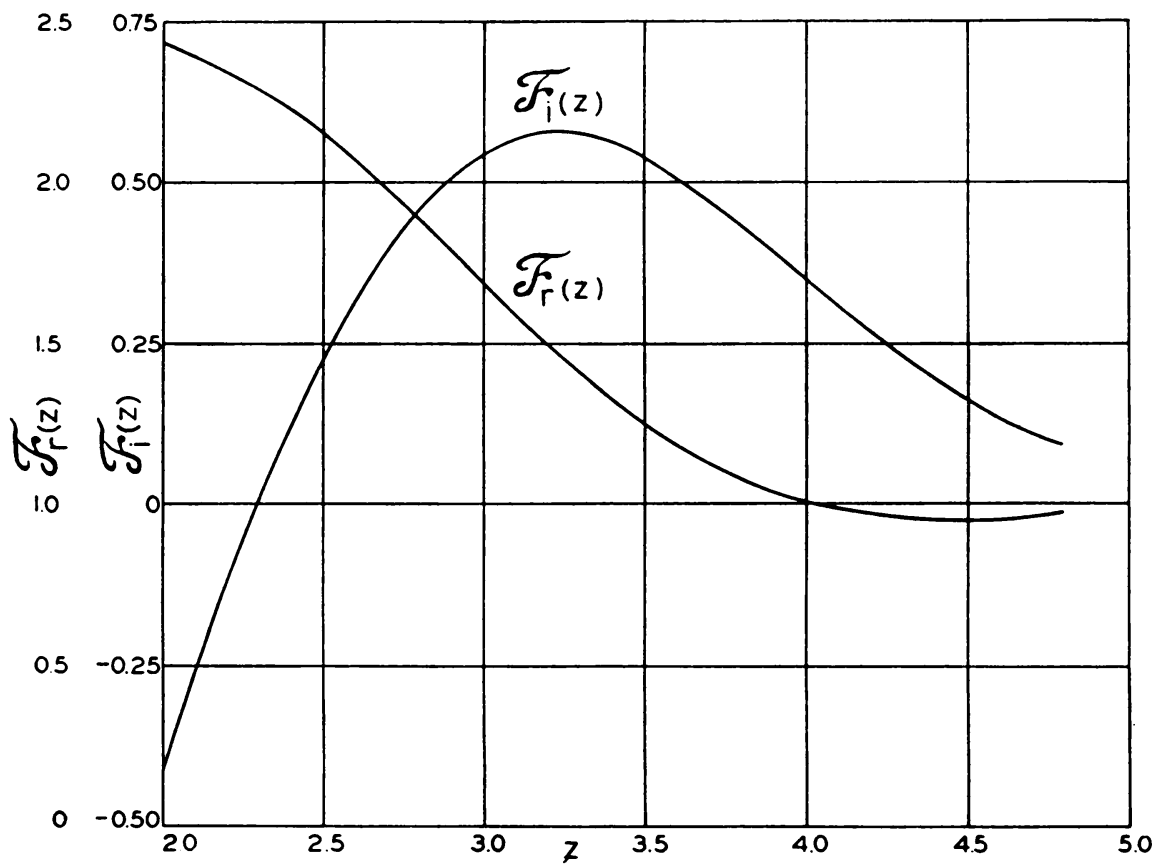

FIg. 4. The function $\mathcal{F}(z)$ shown in its teal and imaginary parts (cf. Table I).

TABLE 1.-The functions $F(z)$ and $\mathcal{f}(z)$.

\begin{tabular}{c|c|c|c|c}
\hline \hline$z$ & $F_{r}$ & $F_{i}$ & $\mathcal{F}_{r}$ & $\mathcal{F}_{i}$ \\
\hline 1.0 & 0.89161 & -0.35025 & 0.80630 & -2.60557 \\
1.2 & 0.78969 & -0.27310 & 1.77012 & -2.29854 \\
1.4 & 0.71970 & -0.21213 & 2.26836 & -1.71669 \\
1.6 & 0.66931 & -0.16009 & 2.44985 & -1.18600 \\
1.8 & 0.63143 & -0.11274 & 2.48104 & -0.75892 \\
2.0 & 0.60144 & -0.06741 & 2.43927 & -0.41253 \\
2.2 & 0.57599 & -0.02226 & 2.35196 & -0.12348 \\
2.4 & 0.55230 & -0.02395 & 2.22724 & 0.11916 \\
2.6 & 0.52773 & -0.07203 & 2.06929 & 0.31558 \\
2.8 & 0.49952 & 0.12220 & 1.88566 & 0.46043 \\
3.0 & 0.46456 & 0.17391 & 1.68938 & 0.54872 \\
3.2 & 0.41947 & 0.22520 & 1.49726 & 0.58082 \\
3.4 & 0.36110 & 0.27193 & 1.32516 & 0.56401 \\
3.6 & 0.28802 & 0.30705 & 1.18429 & 0.51074 \\
3.8 & 0.20352 & 0.32130 & 1.07982 & 0.43560 \\
4.0 & 0.11800 & 0.30721 & 1.01118 & 0.35220 \\
4.2 & 0.04698 & 0.26559 & 0.97361 & 0.27133 \\
4.4 & 0.00240 & 0.20811 & 0.96056 & 0.20038 \\
4.6 & 0.02160 & 0.14475 & 0.95989 & 0.13601 \\
4.8 & 0.01477 & 0.09875 & 0.97659 & 0.09503 \\
\hline
\end{tabular}




\section{REFERENCES}

1. A. B. Bassett, Proc. Roy. Soc. London (A) 52, 273-276 (1893).

2. H. Bateman, Bull. Nat. Research Council, Washington, no. 84, 372-384 (1932).

3. O. Blumenthal, Sitz. Akad. Wiss., München, 563-595 (1913).

4. H. Faxen, Arkiv Mat. Ast. Fysik 21 A, no. 26, 11 p. (1928). (Curved.)

5. K. O. FrIEDRICHS, Fluid dynamics (mimeographed lecture notes, Brown University, 1942), ch. 4, pp. 200-209.

6. S. Goldstein, Proc. Camb. Phil. Soc. 32, 40-54 (1936).

7. S. Goldstrin, Modern developments in fluid dynamics, The Clarendon Press, Oxford, 1938, pp. $135-136,157,194-200,564$.

8. H. Görtler, Zeit. angew. Math. Mech. 20, 138-147 (1940). (Curved.)

9. H. Görtler, Nachr. Ges. Wiss. Göttingen 2, 1-26 (1940). (Curved.)

10. G. Hamel, Nachr. Ges. Wiss. Göttingen, 261-270 (1911).

11. W. H. Harrison, Proc. Camb. Phil. Soc. 20, 455-459 (1920-1921).

12. O. Haupt, Sitz. Akad. Wiss., München, 289 (1912).

13. T. H. Havelock, Proc. Roy. Soc. London (A) 98, 428-437 (1921).

14. W. Heisenberg, Ann. d. Phys. 74, 577-627 (1924).

15. S. H. Hollingdale, Phil. Mag. (7) 29, 209-257 (1940).

16. L. Hopf, Ann. d. Phys. 4, 1-60 (1914).

17. H. Jefrereys, Proc. London Math. Soc. (2) 23, 428-436 (1924).

18. Th. von Kármán, Proc. 1st.Int. Congress Appl. Mech., Delft, 1924, p. 97; Abh. Aerodynam.

Inst. Tech. Hochschule, Aachen, 4, (1925).

19. Th. von Kármán, Proc. 4th Int. Congress Appl. Mech., Cambridge, England, 1934, Cambridge University Press, Cambridge, 1935, pp. 54-60, 77.

20. LoRd Kelvin, Mathematical and physical papers, Cambridge University Press, vol. 4, pp. 186187 (1880).

21. LORD Kelvin, Mathematical and physical papers, Cambridge University Press, vol. 4, pp. 321330, 330-337 (1887).

22. L. V. KING, Phil. Mag. (6) 31, 332-338 (1916).

23. H. A. Kramers, Zeit. f. Phys. 39, 828-836 (1926).

24. R. E. LANGER, Trans. Amer. Math. Soc. 33, 23-64 (1931); 34, 447-480 (1932).

25. R. E. LANGer, Bull. Amer. Math. Soc. 46, 257-263 (1940).

26. L. Lichtenstein, Hydromechanik, Julius Springer, Berlin, 1929, pp. 409-414.

27. C. C. Lin, Proc. Nat. Acad. Sci. U.S.A. 30, 316-324 (1944).

28. J. W. Lewis, Proc. Roy. Soc. London (A) 117, 388-407 (1928). (Curved.)

29. L. A. Lorentz, Abh. theoret. Phys. (Leipzig) 1, 43-71 (1907).

30. W. T. MacCreadie, Proc. Nat. Acad. Sci. U.S.A. 17, 381-388 (1931).

31. R. von Mises, Deut. Math. Ver. 21, 241-248 (1912).

32. R. von Mises, Festschrift Heinrich Weber, Teubner, Leipzig and Berlin, 1912, pp. 252-282.

33. F. Noether, Sitz. Akad. Wiss., München, 309-329 (1913).

34. F. Noether, Deut. Math. Ver. 23, 138-144 (1914).

35. F. Noether, Zeit. angew. Math. Mech. 1, 125-138, 218-219 (1921).

36. F. Noether, Zeit. angew. Math. Mech. 6, 232-243, 339-340, 428, 497-498 (1926).

37. W. M. F. ORR, Proc. Roy. Irish Acad. 27, 9-26, 69-138 (1906-1907).

38. C. W. Oseen, Arkiv. Mat. Ast. Fysik 7, no. 15, 20 p. (1911).

39. C. L. Pekeris, Proc. Cambridge Phil. Soc. 32, 55-66 (1936).

40. C. L. Pekeris, Jour. Aero. Sci. 5, 237-240 (1938).

41. L. Prandtl, Zeit. angew. Math. Mech. 1, 431-436 (1921); 11, 407 (1931); Phys. Zeit. 23, 19-23 (1922); Vorträge aus dem Gebeite der Aerodynamik und Verwandter Gebeite, Aachen, 1929, Julius Springer, Berlin, 1930, pp. 1-7.

42. L. Prandt, Aerodynamic theory, edited by W. F. Durand, Julius Springer, Berlin, 1935, vol. 3, pp. $178-190$.

43. LoRd Rayleigh, Scientific papers, Cambridge University Press, Cambridge, vol. 1, pp. 474-487 (1880); vol. 3, pp. 17-23 (1887); vol. 3, pp. 575-584 (1892); vol. 4, pp. 203-209 (1895); vol. 6, p. 917 (1913).

44. LoRd Rayleigh, Scientific papers, Cambridge University Press, Cambridge, vol. 6, p. 266 (1914); vol. 6, p. 341 (1915). 
45. LoRd Rayleigh, Scientific papers, Cambridge University Press, Cambridge, vol. 6, p. 447 (1916). (Curved.) (1895).

46. O. Reynolds, Scientific papers, Cambridge University Press, Cambridge, vol. 2, pp. 535-577

47. P. Savic, Phil. Mag. (7) 32, 245-252 (1941).

48. P. Savic and J. W. Murphy, Phil. Mag. (7) 34, 139-144 (1943).

49. H. Schlichting, Ann. d. Phys. (5) 14, 905-936 (1932).

50. H. Schlichting, Nachr. Ges. Wiss. Göttingen, 160-198 (1932). (Rotating cylinders.)

51. H. Schlichting, Zeit. angew. Math. Mech. 13, 171-174 (1933).

52. H. Schlichting, Nachr. Ges. Wiss. Göttingen, 181-208 (1933).

53. H. Schlichting, Naturwissenschaften 22, 376-381 (1934).

54. H. Schlichting, Nachr. Ges. Wiss. Göttingen 1, 47-78 (1935).

55. Th. SeXl, Ann. Phys. 83, 835-848 (1927); 84, 807-822 (1927). (Circular tube.)

56. F. R. Sharpe, Trans. Amer. Math. Soc. 6, 496-503 (1905).

57. H. Solberg, Proc. 1st Int. Congress Appl. Mech., Delft, 1924, pp. 387-394.

58. A. Sommerfeld, Proc. 4th Int. Congress Math., Rome, 1908, pp. 116-124.

59. R. V. Southwell and L. Chitty, Trans. Roy. Soc. London (A) 229, 205-253 (1930); Aeronautical Research Committee R. and M. no. 1200, (1930).

60. H. B. SQuire, Proc. Roy. Soc. London (A) 142, 621-628 (1933).

61. J. L. Synge, Trans. Roy. Soc. Canada 27, 1-18 (1933).

62. J. L. Synge, J. Math. Physics 15, 205-210 (1936).

63. J. L. Synge, Semicentennial publications of the Amer. Math. Soc., 1938, vol. 2, pp. 227-269.

64. J. L. Synge, Proc. 5th Int. Congress Appl. Mech., Cambridge, Mass., U.S.A., 1938, Wiley and Sons, New York, 1939, pp. 326-332.

65. J. L. Synge, Proc. Roy. Soc. London (A) 167, 250-256 (1938). (Curved.)

66. K. Tamaki and W. J. Harrison, Trans. Cambridge Phil. Soc. 22, 425-438 (1920). (Curved.)

67. G. I. TAYLor, Phil. Trans. Roy. Soc. London (A) 223, 289-343 (1923); also, Proc. 1st Int. Congress Appl. Mech. Delft, 1924, p. 89. (Curved.)

68. G. I. TAYLOR, Proc. Roy. Soc. London (A) 156, 307-317 (1936).

69. G. I. TAYLOR, Proc. Roy. Soc. London (A) 157, 546-564 (1936).

70. G. I. TAYLOR, Proc. 5th Int. Congress Appl. Mech., Cambridge, Mass., U.S.A., 1938, Wiley and Sons, New York, 1939, pp. 304-310.

71. T. Y. Thомas, Amer. J. Math. 64, 754-767 (1942); Proc. Nat. Acad. Sci. U.S.A. 29, 243-246 (1943).

72. O. Teitjens, Zeit. angew. Math. Mech. 5, 200-217 (1925).

73. W. Tollmien, Nachr. Ges. Wiss. Göttingen, 21-44 (1929); Proc. 3rd Int. Congress Appl. Mech., Stockholm, 1930, vol. 1, pp. 105-108; Vorträge aus dem Gebeite der Aerodynamik und verwandte Gebeite, Aachen, 1929, Julius Springer, Berlin, 1930, pp. 18-21.

74. W. Tollmien, Nachr. Ges. Wiss. Göttingen (Neue Folge) 1, 70-114 (1935).

75. W. Tollmien, Zeit. angew. Math. Mech. 15, 96-100 (1935).

76. G. N. Watson, Theory of Bessel functions, Cambridge University Press, Cambridge, ed. 1, 1922, or ed. 2, 1944, p. 198; for Stokes' phenomenon, see p. 201. 